TRANSACTIONS OF THE

AMERICAN MATHEMATICAL SOCIETY

Volume 357, Number 3, Pages 965-981

S 0002-9947(04)03687-6

Article electronically published on October 19, 2004

\title{
RESULTANTS AND DISCRIMINANTS OF CHEBYSHEV AND RELATED POLYNOMIALS
}

\author{
KARL DILCHER AND KENNETH B. STOLARSKY
}

\begin{abstract}
We show that the resultants with respect to $x$ of certain linear forms in Chebyshev polynomials with argument $x$ are again linear forms in Chebyshev polynomials. Their coefficients and arguments are certain rational functions of the coefficients of the original forms. We apply this to establish several related results involving resultants and discriminants of polynomials, including certain self-reciprocal quadrinomials.
\end{abstract}

\section{INTRODUCTION}

The resultant of two polynomials is in general a rather complicated function of their coefficients. However, there is an elegant formula (see Section 2) for the resultant of any two cyclotomic polynomials. The books [14] and [17] establish the well-known formulas for discriminants of Chebyshev polynomials, but do not discuss their resultants. We establish here, in Theorem 2, a formula for the resultants of certain linear forms in Chebyshev polynomials of the second kind. It is perhaps surprising in that it shows such resultants to be themselves simply expressible in terms of Chebyshev polynomials whose coefficients and arguments are rational functions of the coefficients of the forms. Next, there is a compact elegant formula for the discriminant of a general trinomial (e.g., [16], p. 1105 or [7], p. 406), but no such formula seems to exist for the general quadrinomial. Our remaining investigations into resultants and discriminants of polynomials related to those considered above include a "Chebyshevian" formula for the discriminants of certain self-reciprocal quadrinomials. It is similar to (but somewhat more intricate than) the result of Theorem 2.

Our approach throughout is to rely upon algebraic properties of the resultant, especially with respect to the division and Euclidean algorithms for polynomials. This is in the spirit of [16], in which there is a very readable account of the proof of the trinomial discriminant formula. (The alternative derivation of this formula by using properties of determinants is given in 6].) In Lemma 6.3 we show that the explicit form of the quotients for the relevant Euclidean algorithm involves a remarkable product of fourth powers of linear forms in Chebyshev polynomials.

Received by the editors November 1, 2002.

2000 Mathematics Subject Classification. Primary 12E10, 12E05; Secondary 13P05, 33C45.

Key words and phrases. Resultants, discriminants, Chebyshev polynomials, cyclotomic polynomials.

This research was supported in part by the Natural Sciences and Engineering Research Council of Canada. 
In Section 2 we introduce the polynomials to be studied and state our main results on resultants. The corresponding results on discriminants are stated in Section 3. We recall the most important properties of resultants and discriminants in Section 4, and then prove our results in Sections 5 and 6. In Section 7 we give some simple consequences of our results that help to put them into perspective.

\section{Motivation And Results}

The cyclotomic polynomial $\Phi_{n}(x)$ is the unique monic polynomial whose roots are the primitive $n$th roots of unity. Therefore it has degree $\varphi(n)$ and can be written as

$$
\Phi_{n}(x)=\prod_{\substack{k=1 \\(k, n)=1}}^{n}\left(x-e^{2 \pi i k / n}\right) .
$$

Also, it has integer coefficients and is irreducible over $\mathbb{Q}$.

Although partial results on the resultant of two cyclotomic polynomials had been known before, the first complete treatment appears in [3]. The main results can be summarized as follows:

Theorem 1 (Apostol). For $m>n>1$ we have

$$
\operatorname{Res}\left(\Phi_{m}, \Phi_{n}\right)= \begin{cases}p^{\varphi(n)} & \text { if } \frac{m}{n} \text { is a power of a prime } p \\ 1 & \text { otherwise. }\end{cases}
$$

Apostol himself subsequently introduced parameters and extended this result to $\operatorname{Res}\left(\Phi_{m}(a x), \Phi_{n}(b x)\right)$; see [4].

We are now going to introduce parameters in a different way. By the well-known relation

$$
x^{N}-1=\prod_{d \mid N} \Phi_{d}(x)
$$

and by Theorem 1 we see that the resultant of the polynomials

$$
\frac{x^{2 n+2}-1}{x^{2}-1}, \quad \frac{x^{2 n}-1}{x^{2}-1}
$$

is always 1 . We now change the numerators to a closely related quadrinomial which is of interest also in different connections; we define

$$
f_{n, k}(x):=x^{2 n}+k x^{2 n-1}-k x-1
$$

and

$$
q_{n, k}(x):=\frac{f_{n, k}(x)}{x^{2}-1}=x^{2 n-2}+k x^{2 n-3}+x^{2 n-4}+\ldots+x^{2}+k x+1
$$

and consider the resultant

$$
r_{n}(h, k):=\operatorname{Res}\left(q_{n+1, k}, q_{n, h}\right) .
$$


We know that $r_{n}(0,0)=1$; the question arises as to what $r_{n}$ is as a function of $h$ and $k$. Experimentation with computer algebra yields

$$
\begin{aligned}
& r_{0}(h, k)=r_{1}(h, k)=1, \\
& r_{2}(h, k)=\left(1+h k-h^{2}\right)^{2}, \\
& r_{3}(h, k)=\left(1+2 h k-2 h^{2}+h^{2} k^{2}-h^{3} k\right)^{2}, \\
& r_{4}(h, k)=\left(1+3 h k-3 h^{2}+3 h^{2} k^{2}-4 h^{3} k+h^{3} k^{3}-h^{4} k^{2}+h^{4}\right)^{2} .
\end{aligned}
$$

This leads us to conjecture that the resultants are always squares of polynomials $d_{n}(h, k)$ in the two variables $h, k$ and, upon closer inspection, that these polynomials satisfy the recurrence relation

$$
d_{0}(x, y)=d_{1}(x, y)=1,
$$

and

$$
d_{n+1}(x, y)=(1+x y) d_{n}(x, y)-x^{2} d_{n-1}(x, y) .
$$

Rewriting this as

$$
x^{-(n+1)} d_{n+1}(x, y)=2 \frac{1+x y}{2 x} x^{-n} d_{n}(x, y)-x^{-(n-1)} d_{n-1}(x, y),
$$

we observe that the polynomials $x^{-n} d_{n}(x, y)$ satisfy the same recurrence relation as the Chebyshev polynomial of the second kind $U_{n}(z)$, with $z=(1+x y) / 2 x$. The $U_{n}(z)$ can be defined by (see, e.g., [14] or [1, Ch. 22)

$$
U_{0}(z)=1, \quad U_{1}(z)=2 z, \quad \text { and } \quad U_{n+1}(z)=2 z U_{n}(z)-U_{n-1}(z) .
$$

Setting up $x^{-n} d_{n}(x, y)$ as a linear combination of two successive Chebyshev polynomials of the second kind, we easily find the conjectured form

$$
d_{n}(x, y)=x^{n}\left[U_{n}\left(\frac{1+x y}{2 x}\right)-y U_{n-1}\left(\frac{1+x y}{2 x}\right)\right] .
$$

Another connection with the Chebyshev polynomials becomes apparent when we rewrite the polynomial $q_{n, k}$ as

$$
q_{n, k}(z)=z^{n-1} \frac{\left(z^{n}-z^{-n}\right)+k\left(z^{n-1}-z^{-(n-1)}\right)}{z-z^{-1}} .
$$

We set $z=e^{i \theta}$ and use the fact that

$$
U_{n-1}(\cos \theta)=\frac{\sin n \theta}{\sin \theta} \text {. }
$$

Then with $2 \cos \theta=z+1 / z$ and $2 i \sin \theta=z-1 / z$, and upon replacing $n$ by $n+1$ and $z$ by $x$, we obtain

$$
q_{n+1, k}(x)=x^{n}\left[U_{n}\left(\frac{1}{2}\left(x+\frac{1}{x}\right)\right)+k U_{n-1}\left(\frac{1}{2}\left(x+\frac{1}{x}\right)\right)\right] .
$$

This particular form of the polynomial $q_{n, k}$ motivates our main result:

Theorem 2. For $n \geq 2$ we have

$$
\operatorname{Res}\left(U_{n}(x)+k U_{n-1}(x), U_{n-1}(x)+h U_{n-2}(x)\right)=(-1)^{\frac{n(n-1)}{2}} 2^{n(n-1)} d_{n}(h, k) \text {, }
$$

where the polynomial $d_{n}(h, k)$ is as defined in (2.8).

We defer the proof of this theorem to Section 6 .

As a first application we obtain the conjectured evaluation of the resultant $r_{n}(h, k)$ defined in (2.4); the proof will be given in Section 5 . 
Theorem 3. For $n \geq 0$ we have

$$
\operatorname{Res}\left(q_{n+1, k}(x), q_{n, h}(x)\right)=d_{n}(h, k)^{2},
$$

where the polynomials $q_{n, k}(x)$ and $d_{n}(h, k)$ are as defined in (2.3), resp. (2.5).

\section{DisCRIMINANTS}

We now turn to the discriminant to obtain further surprising results and interconnections concerning the various polynomials introduced above.

From a detailed analysis, done elsewhere, of the quadrinomial $x^{m}+k x^{m-1}-k x-1$ it follows that the value $k=m /(m-2)$ plays a special role. In fact, for this value of $k$ all the zeros of the quadrinomial lie on the unit circle, and it is easy to verify that it has a simple zero at $x=1$ and a triple zero at $x=-1$. The discriminant of the quadrinomial will therefore vanish for $k=m /(m-2)$. As we did with the resultant (2.4), we can ask how the discriminant behaves as a function of $k$. We restrict our attention to the more interesting case of even $m=2 n$, i.e., we will consider the quadrinomial $f_{n, k}(x)$ defined in (2.2). Computer algebra suggests that the discriminant always has a factor of the form

$$
(n-(n-1) k)^{3}(n+(n-1) k)^{3},
$$

times the square of a polynomial $a_{n-2}(k)$, where the first few terms of this polynomial sequence are

$$
\begin{aligned}
& a_{0}(k)=1, \\
& a_{1}(k)=k^{2}+4, \\
& a_{2}(k)=4 k^{4}+13 k^{2}+32, \\
& a_{3}(k)=32 k^{6}+93 k^{4}+204 k^{2}+400, \\
& a_{4}(k)=400 k^{8}+1084 k^{6}+2213 k^{4}+4032 k^{2}+6912, \\
& a_{5}(k)=6912 k^{10}+17856 k^{8}+34709 k^{6}+60152 k^{4}+98000 k^{2}+153664, \\
& a_{7}(k)=153664 k^{12}+\cdots+4194304 .
\end{aligned}
$$

Observe that the constant term of $a_{n-1}(k)$ coincides with the leading coefficient of $a_{n}(k)$. The above is the content of Theorem 5 and Corollary 3.2.

We begin with a result that is analogous to Theorem 2 in that it implies all further discriminant results in this section. We follow Rivlin in [14 in letting $D_{x}(f)$ denote the discriminant with respect to $x$ of a polynomial $f$. For the precise normalization of the discriminant, its relationship to the resultant, and a few of its properties that will be useful here, see Lemma 4.3 and the paragraph preceding it.

Theorem 4. For all $n \geq 1$ we have

$$
D_{x}\left(U_{n}(x)+k U_{n-1}(x)\right)=2^{n(n-1)} a_{n-1}(k),
$$

where

$$
a_{n-1}(k)=(-1)^{n} \frac{(2 n+1)^{n} k^{n}}{(n+1)^{2}-n^{2} k^{2}}\left[U_{n}\left(-\frac{n+1+n k^{2}}{(2 n+1) k}\right)+k U_{n-1}\left(-\frac{n+1+n k^{2}}{(2 n+1) k}\right)\right]
$$

is an even polynomial in $k$ of degree $2 n-2$ with positive integer coefficients.

It will become clear from the next result that this is the polynomial of (3.1). 
Theorem 5. Let $f_{n, k}(x)$ be as in (2.2). For $n \geq 2$ we have

$$
D_{x}\left(f_{n, k}\right)=4(-1)^{n-1}\left(n^{2}-(n-1)^{2} k^{2}\right)^{3} a_{n-2}(k)^{2},
$$

with the polynomial $a_{n}(k)$ as in (3.3).

Next we will see that this polynomial $a_{n}(x)$ occurs, even twice, in connection with another polynomial of Section 2.

Theorem 6. Let $d_{n}(x, y)$ be as in (2.5) or (2.8). Then for $n \geq 2$ we have

$$
\begin{aligned}
& D_{x}\left(d_{n}(x, y)\right)=a_{n-1}(y), \\
& D_{y}\left(d_{n}(x, y)\right)=x^{2(n-1)(n-2)} a_{n-2}(x) .
\end{aligned}
$$

The following lemma, which will be needed in the proof of Theorem 5, is also of interest here.

Lemma 3.1. Let $q_{n, k}(x)$ be as in (2.3). For $n \geq 1$ we have

$$
D_{x}\left(f_{n, k}\right)=4\left(n^{2}-(n-1)^{2} k^{2}\right)^{2} D_{x}\left(q_{n, k}\right) .
$$

This implies immediately:

Corollary 3.1. For $n \geq 2$ we have

$$
D_{x}\left(q_{n, k}\right)=(-1)^{n-1}\left(n^{2}-(n-1)^{2} k^{2}\right) a_{n-2}(k)^{2} .
$$

If we compare this corollary with Theorems 3 and 6 , we see that, up to certain "easy" factors, the square root of the discriminant of the polynomial $q_{n, k}$ is the same as the discriminant of the square root of the resultant of two "neighboring" such polynomials.

We conclude this section with some further properties of the polynomial $a_{n-1}(k)$, followed by an application to cyclotomic polynomials.

Corollary 3.2. Let $a_{n-1}(k)$ be as defined in (3.3). Then the leading coefficient of this polynomial is $2^{n-1} n^{n-3}$, and furthermore

$$
\begin{aligned}
a_{n-1}(0) & =2^{n}(n+1)^{n-2}, \\
a_{n-1}( \pm 1) & =(2 n+1)^{n-1} .
\end{aligned}
$$

Proof. The first statement and (3.9) follow from the fact that $U_{n}(x)$ has leading coefficient $2^{n}$, while (3.10) relies on the special values (see, e.g., [1, p. 777)

$$
U_{n}(1)=n+1, \quad U_{n}(-1)=(-1)^{n}(n+1) .
$$

We now briefly turn to three special cases of cyclotomic polynomials. Let $p$ be an odd prime; it is easy to see (e.g., with (2.1) that

$$
\begin{gathered}
\Phi_{p}(x)=x^{p-1}+x^{p-2}+\cdots+x+1=q_{n, 1}(x), \quad p=2 n-1, \\
\Phi_{2 p}(x)=x^{p-1}-x^{p-2}+\cdots-x+1=q_{n,-1}(x), \quad p=2 n-1, \\
\Phi_{4 p}(x)=x^{2 p-2}-x^{2 p-4}+\cdots-x^{2}+1=q_{n, 0}(i x), \quad p=n,
\end{gathered}
$$

where the second equality in each line is obvious with (2.3). Now it follows immediately from Corollaries 3.1 and 3.2 that

$$
D_{x}\left(\Phi_{p}\right)=D_{x}\left(\Phi_{2 p}\right)=(-1)^{\frac{p-1}{2}} p^{p-2} .
$$


To deal with (3.14), we use the property (4.14) below to get

$$
D_{x}\left(q_{n, 0}(i x)\right)=i^{(2 p-2)(2 p-3)} D_{x}\left(q_{n, 0}(x)\right)=(-1)^{p-1} D_{x}\left(q_{n, 0}(x)\right),
$$

and thus, again with Corollaries 3.1 and 3.2,

$$
D_{x}\left(\Phi_{4 p}\right)=2^{2 p-2} p^{2 p-4} .
$$

This method could be used to cover more classes of cyclotomic polynomials. However, a general formula has long been known (see [13]): If $n=p_{1}^{\alpha_{1}} \cdot \ldots \cdot p_{s}^{\alpha_{s}}$, where $p_{1}, \ldots, p_{s}$ are distinct primes, then

$$
D_{x}\left(\Phi_{n}\right)=\frac{(-1)^{\varphi(n) / 2} n^{\varphi(n)}}{p_{1}^{\varphi(n) / \varphi\left(p_{1}\right)} \cdot \ldots \cdot p_{s}^{\varphi(n) / \varphi\left(p_{s}\right)}} .
$$

The above remarks show that (3.8) can be considered an interpolating formula for discriminants of certain cyclotomic polynomials, in a similar way as Theorem 3 relates to the resultants of cyclotomic polynomials.

\section{Some PROperties of RESUltants AND Discriminants}

In this section we quote only those properties that are used in the proofs below. For the basic theory see, e.g., [18]; a more complete treatment can be found in [7, Ch. 12. Some relatively recent developments are discussed in [9], and discriminants of generalized Chebyshev polynomials are studied in [15]; there appears to be no overlap with our work. For discriminants from a special functions point of view, see [17], 8], or [2], Ch. 8, especially Section 8.5. Let

$$
\begin{aligned}
& f(x)=a_{0} x^{n}+a_{1} x^{n-1}+\cdots+a_{n-1} x+a_{n}, \\
& g(x)=b_{0} x^{m}+b_{1} x^{m-1}+\cdots+b_{m-1} x+b_{m}
\end{aligned}
$$

be two given polynomials. Then the resultant of $f$ and $g$ is usually defined by the Sylvester determinant, the determinant of an $(m+n) \times(m+n)$ matrix which has the coefficients of $f$ and $g$ as entries; see, e.g., 3], [10, [7], 12], or [18. We denote the resultant of $f$ and $g$ by $\operatorname{Res}_{x}(f, g)$, or simply $\operatorname{Res}(f, g)$ if there is no ambiguity as to the unknown with respect to which the resultant is taken.

Suppose that the zeros of $f$ and $g$ are $\alpha_{1}, \ldots, \alpha_{n}$ and $\beta_{1}, \ldots, \beta_{m}$, respectively. Then the most important properties are

$$
\begin{array}{r}
\operatorname{Res}(f, g)=a_{0}^{m} b_{0}^{n} \prod_{i=1}^{n} \prod_{j=1}^{m}\left(\alpha_{i}-\beta_{j}\right), \\
\operatorname{Res}(f, g)=a_{0}^{m} \prod_{i=1}^{n} g\left(\alpha_{i}\right), \\
\operatorname{Res}(f, g)=(-1)^{n m} \operatorname{Res}(g, f), \\
\operatorname{Res}(f, p q)=\operatorname{Res}(f, p) \operatorname{Res}(f, q) .
\end{array}
$$

Furthermore, if $a$ is a constant then, unless $a=g=0$,

$$
\operatorname{Res}(a, g)=\operatorname{Res}(g, a)=a^{\operatorname{deg} g}
$$

and if $a, b$ are constants, then

$$
\operatorname{Res}(a, b)=1
$$


Most of these properties can be found in the references mentioned above; particular care was taken in [10] in the cases where one or both of the polynomials was a constant or zero. We state two other useful properties as a lemma.

Lemma 4.1. Let the polynomials $f$ and $g$ be as in (4.1).

(a) If we can write

$$
f(x)=q(x) g(x)+r(x),
$$

with polynomials $q, r$, and $\nu:=\operatorname{deg} r$, then

$$
\operatorname{Res}(g, f)=b_{0}^{n-\nu} \operatorname{Res}(g, r) .
$$

(b) If $\operatorname{deg}(q f+g)=\operatorname{deg} g$ for a polynomial (or constant) $q$, then

$$
\operatorname{Res}(f, q f+g)=\operatorname{Res}(f, g) .
$$

Proof. (a) By (4.3) we have

$$
\operatorname{Res}(g, f)=b_{0}^{n} \prod_{j=1}^{m} f\left(\beta_{j}\right)
$$

and similarly,

$$
\operatorname{Res}(g, r)=b_{0}^{\nu} \prod_{j=1}^{m} r\left(\beta_{j}\right)=b_{0}^{\nu} \prod_{j=1}^{m}\left(f\left(\beta_{j}\right)-q\left(\beta_{j}\right) g\left(\beta_{j}\right)\right)=b_{0}^{\nu} \prod_{j=1}^{m} f\left(\beta_{j}\right),
$$

and this, with (4.11) gives (4.9).

(b) We apply part (a) to $g(x)=(-q(x)) f(x)+(q f+g)(x)$. Then by the condition of (b) we have $n=\nu$, and (4.10) follows from (4.9).

We remark that part (a) is stated in [12, p. 58, for the case $\nu<m \leq n$; the proof, as indicated there, is identical with ours. A special case is also given in [5]. The identity (4.9) is important for the evaluation of resultants, especially when (4.8) represents the division algorithm (see 12, p. 58). It should be noted that the convention concerning the sign of the resultant differs between [12] and the other publications cited.

We will also use the resultant of two homogeneous polynomials

$$
\begin{aligned}
& F(x, y)=a_{0} x^{n}+a_{1} x^{n-1} y+\cdots+a_{n-1} x y^{n-1}+a_{n} y^{n}, \\
& G(x, y)=b_{0} x^{m}+b_{1} x^{m-1} y+\cdots+b_{m-1} x y^{m-1}+b_{m} y^{m} .
\end{aligned}
$$

The resultant of $F$ and $G$, denoted by $\operatorname{Res}_{x, y}(F, G)$, is defined by the same Sylvester determinant. The basic properties are identical or analogous to those of $\operatorname{Res}(f, g)$; for details, see [11]. Also in [11, the following interesting and useful "chain rule" for resultants of homogeneous polynomials is derived:

Lemma 4.2 (McKay and Wang). Let $F(x, y), G(x, y), H(u, v)$, and $K(u, v)$ be homogeneous polynomials of degrees at least 1 , and suppose that $\operatorname{deg} H=\operatorname{deg} K$. Then

$$
\operatorname{Res}_{u, v}(F(H, K), G(H, K))=\left(\operatorname{Res}_{x, y}(F, G)\right)^{\operatorname{deg} H}\left(\operatorname{Res}_{u, v}(H, K)\right)^{\operatorname{deg} F \operatorname{deg} G} .
$$

Some additional cases, where some of the polynomials are constant or zero, are also treated in [11]. An analogous result for nonhomogeneous polynomials can be found in [10]. 
The discriminant $D_{x}(f)$ of a polynomial $f$ (as above) can be given in terms of a resultant by

$$
D_{x}(f)=\frac{1}{a_{0}}(-1)^{\frac{n(n-1)}{2}} \operatorname{Res}\left(f, f^{\prime}\right),
$$

where $f^{\prime}$ is the derivative of $f$. For standard properties of the discriminant see, e.g., any of the references cited above in connection with resultants. It should be noted that occasionally the discriminant is defined without the powers of -1 in (4.13). The following properties will be needed below in Section 5; we were unable to find them explicitly stated in the literature.

Lemma 4.3. Let $f(x)=a_{0} x^{n}+a_{1} x^{n-1}+\cdots+a_{n}$ be a polynomial, and $a, b, c$ real constants. Then

$$
\begin{aligned}
D_{x}(f(a x+b)) & =a^{n(n-1)} D_{x}(f(x)), \\
D_{x}(c f(x)) & =c^{2(n-1)} D_{x}(f(x)) .
\end{aligned}
$$

Both these identities can be easily obtained with (4.9) and (4.5); the chain rule for resultants (in the form of [10]) is applied in the case of (4.14), and (4.6), (4.7) are used for (4.15).

\section{Proofs of Theorems $3-6$}

In this section we will prove Theorems 3-6 and Lemma 3.1. Some of these proofs rely on Theorem 2 which will be proved later, in Section 6 .

Proof of Theorem 3. For $n=0,1$ this can be verified by direct calculation. For $n \geq 2$ we use (2.10), and we deal with the polynomials (2.9) by applying Lemma 4.2. We set

$$
\begin{aligned}
& f(w):=U_{n}(w)+k U_{n-1}(w)=f_{0} w^{n}+f_{1} w^{n-1}+\cdots+f_{n}, \\
& g(w):=U_{n-1}(w)+h U_{n-2}(w)=g_{0} w^{n-1}+g_{1} w^{n-2}+\cdots+g_{n-1} .
\end{aligned}
$$

Then we define the homogeneous polynomials $F, G$ in two variables by

$$
\begin{aligned}
(2 z)^{n} f\left(\frac{z^{2}+1}{2 z}\right) & =f_{0} \cdot\left(z^{2}+1\right)^{n}+f_{1} \cdot\left(z^{2}+1\right)^{n-1}(2 z)+\cdots+f_{n} \cdot(2 z)^{n} \\
& =F\left(z^{2}+1,2 z\right), \\
(2 z)^{n-1} g\left(\frac{z^{2}+1}{2 z}\right) & =g_{0} \cdot\left(z^{2}+1\right)^{n-1}+g_{1} \cdot\left(z^{2}+1\right)^{n-2}(2 z)+\cdots+g_{n-1} \cdot(2 z)^{n-1} \\
& =G\left(z^{2}+1,2 z\right) .
\end{aligned}
$$

The polynomials $z^{2}+1$ and $2 z$ can also be changed into homogeneous polynomials of equal degrees, namely

$$
H(z, u):=z^{2}+u^{2}, \quad K(z, u):=2 z u .
$$

We now use (4.12) to obtain

$$
\begin{aligned}
& \operatorname{Res}_{z}\left(F\left(z^{2}+1,2 z\right), G\left(z^{2}+1,2 z\right)\right)=\operatorname{Res}_{z, u}(F(H, K), G(H, K)) \\
& =\left[\operatorname{Res}_{x, y}(F(x, y), G(x, y))\right]^{2}\left[\operatorname{Res}_{z, u}(H(z, u), K(z, u))\right]^{n(n-1)} \\
& =\left[\operatorname{Res}_{x}(F(x, 1), G(x, 1))\right]^{2}\left[\operatorname{Res}_{z}(H(z, 1), K(z, 1))\right]^{n(n-1)} \\
& =\left[(-1)^{n(n-1) / 2} 2^{n(n-1)} d_{n}(h, k)\right]^{2} 4^{n(n-1)}=2^{4 n(n-1)} d_{n}(h, k)^{2},
\end{aligned}
$$


where the first resultant in the third row is just (2.10), and the second resultant is easy to compute directly.

Next we use (2.9) and the properties (4.5), (4.6), and (4.7) to find

$$
\begin{aligned}
& \operatorname{Res}_{z}\left(F\left(z^{2}+1,2 z\right), G\left(z^{2}+1,2 z\right)\right)=\operatorname{Res}_{z}\left(2^{n} q_{n+1, k}(z), 2^{n-1} q_{n, h}(z)\right) \\
& =\operatorname{Res}_{z}\left(2^{n}, 2^{n-1}\right) \cdot \operatorname{Res}_{z}\left(2^{n}, q_{n, h}\right) \cdot \operatorname{Res}_{z}\left(q_{n+1, k}, 2^{n-1}\right) \cdot \operatorname{Res}_{z}\left(q_{n+1, k}, q_{n, h}\right) \\
& =1 \cdot\left(2^{n}\right)^{2 n-2} \cdot\left(2^{n-1}\right)^{2 n} \cdot \operatorname{Res}_{z}\left(q_{n+1, k}(z), q_{n, h}(z)\right),
\end{aligned}
$$

where we have used the fact that $\operatorname{deg} q_{n, h}(z)=2 n-2$. This, combined with the previous string of equations, proves the identity (2.11).

Proof of Theorem 4. To simplify notation, we set

$$
V_{n}(x):=U_{n}(x)+k U_{n-1}(x) .
$$

To deal with the derivative of $V_{n}(x)$, we use the identity

$$
\left(1-x^{2}\right) \frac{d}{d x} U_{n}(x)=-n x U_{n}(x)+(n+1) U_{n-1}(x)
$$

(see, e.g., [1], p. 783). With this and the recurrence relation (2.7), used in the form $U_{n-2}(x)=2 x U_{n-1}(x)-U_{n}(x)$, we obtain upon simplification,

$$
\left(1-x^{2}\right) \frac{d}{d x} V_{n}(x)=-n(k+x) U_{n}(x)+(n+1)(k x+1) U_{n-1}(x) .
$$

It is easy to verify that the right-hand side of this last expression is equal to

$$
(-n(k+x))\left(U_{n}(x)+k U_{n-1}(x)\right)+\left((2 n+1) k x+n+1+n k^{2}\right) U_{n-1}(x),
$$

so that

$$
h_{n}(x):=\left(1-x^{2}\right) V_{n}^{\prime}(x)
$$

and

satisfy

$$
s_{n}(x):=(2 n+1) k x+n+1+n k^{2}
$$

We can now apply Lemma 4.1(a) to (5.4), with the role of $n-\nu$ played by $(n+1)-n=1$ and $b_{0}=2^{n}$. This gives

$$
\operatorname{Res}\left(V_{n}(x),\left(1-x^{2}\right) V_{n}^{\prime}(x)\right)=2^{n} \operatorname{Res}\left(V_{n}(x), s_{n}(x) U_{n-1}(x)\right) .
$$

First we deal with the left-hand side of (5.5). By (4.4) and (4.3) we have

$$
\begin{aligned}
\operatorname{Res}\left(V_{n}(x), 1-x^{2}\right) & =\operatorname{Res}\left(1-x^{2}, V_{n}(x)\right)=(-1)^{n} V_{n}(-1) V_{n}(1) \\
& =(-1)^{n}\left(U_{n}(-1)+k U_{n-1}(-1)\right)\left(U_{n}(1)+k U_{n-1}(1)\right),
\end{aligned}
$$

and using the special values (3.11) we immediately get

$$
\operatorname{Res}\left(V_{n}(x), 1-x^{2}\right)=(n+1)^{2}-n^{2} k^{2} .
$$

To deal with the right-hand side of (5.5), we note that by Theorem 2,

$$
\operatorname{Res}\left(V_{n}(x), U_{n-1}(x)\right)=(-1)^{\frac{n(n-1)}{2}} 2^{n(n-1)} d_{n}(0, k) .
$$

Now it follows from (2.8) that $d_{n}(0, k)=1$; this is also clear from (2.5). Hence

$$
\operatorname{Res}\left(V_{n}(x), U_{n-1}(x)\right)=(-1)^{\frac{n(n-1)}{2}} 2^{n(n-1)} .
$$


Next, we need to consider $\operatorname{Res}\left(U_{n}(x)+k U_{n-1}(x),(2 n+1) k x+n+1+n k^{2}\right)$. By (4.4) and (4.3), this expression is equal to

$$
(-1)^{n}((2 n+1) k)^{n}\left[U_{n}\left(-\frac{n+1+n k^{2}}{(2 n+1) k}\right)+k U_{n-1}\left(-\frac{n+1+n k^{2}}{(2 n+1) k}\right)\right] .
$$

This, combined with (4.13), (5.5), (5.6), and (5.7), finally gives (3.3).

Note that the above linear combination of Chebyshev polynomials is indeed a multiple of the denominator polynomial $(n+1)^{2}-n^{2} k^{2}$ in (3.3) since for $k=$ $\pm(n+1) / n$ we have $\left(n+1+n k^{2}\right) /(2 n+1) k= \pm 1$, and with (3.11) it is easy to see that $U_{n}(\mp 1) \pm \frac{n+1}{n} U_{n-1}(\mp 1)=0$. The fact that $a_{n}(k)$ is an even polynomial follows from $U_{n}$ being even or odd, according to the parity of $n$.

Proof of Lemma 3.1. Recall that $f_{n, k}(x)=\left(x^{2}-1\right) q_{n, k}(x)$. By (4.13) we need to evaluate

$$
\operatorname{Res}\left(\left(x^{2}-1\right) q_{n, k}(x), f_{n, k}^{\prime}(x)\right)=\operatorname{Res}\left(x^{2}-1, f_{n, k}^{\prime}(x)\right) \cdot \operatorname{Res}\left(q_{n, k}(x), f_{n, k}^{\prime}(x)\right) .
$$

Since

$$
\frac{d}{d x}\left(\left(x^{2}-1\right) q_{n, k}(x)\right)=2 x q_{n, k}(x)+\left(x^{2}-1\right) q_{n, k}^{\prime}(x),
$$

we have by (4.3),

$$
\begin{aligned}
\operatorname{Res}\left(x^{2}-1, f_{n, k}^{\prime}(x)\right) & =2 \cdot q_{n, k}(1) \cdot(-2) \cdot q_{n, k}(-1) \\
& =-4(n+(n-1) k)(n-(n-1) k),
\end{aligned}
$$

where the second equality is immediate from (2.3). Next, (5.9) and Lemma 4.1(b) give

$$
\begin{aligned}
\operatorname{Res}\left(q_{n, k}(x), f_{n, k}^{\prime}(x)\right) & =\operatorname{Res}\left(q_{n, k}(x),\left(x^{2}-1\right) q_{n, k}^{\prime}(x)\right) \\
& =\operatorname{Res}\left(q_{n, k}(x), x^{2}-1\right) \operatorname{Res}\left(q_{n, k}(x), q_{n, k}^{\prime}(x)\right) .
\end{aligned}
$$

Using (4.4) and once again (4.3), we get

$$
\begin{aligned}
\operatorname{Res}\left(q_{n, k}(x), x^{2}-1\right) & =\operatorname{Res}\left(x^{2}-1, q_{n, k}(x)\right) \\
& =q_{n, k}(1) \cdot q_{n, k}(-1)=n^{2}-(n-1)^{2} k^{2},
\end{aligned}
$$

as in (5.10). Combining this with (5.8), (5.10) and (5.11), we obtain

$$
\operatorname{Res}\left(f_{n, k}(x), f_{n, k}^{\prime}(x)\right)=-4\left(n^{2}-(n-1)^{2} k^{2}\right)^{2} \operatorname{Res}\left(q_{n, k}(x), q_{n, k}^{\prime}(x)\right),
$$

and (4.13) gives (3.7), after a straightforward calculation of the powers of -1 .

Proof of Theorem 5. By Lemma 3.1 and Theorem 4 it suffices to show that

$$
D_{x}\left(q_{n+1, k}(x)\right)=(-1)^{n} 2^{-2 n(n-1)}\left((n+1)^{2}-n^{2} k^{2}\right) D_{x}\left(V_{n}(x)\right)^{2},
$$

with $V_{n}$ as defined in (5.2). We begin with the observations that by (4.13) we have

$$
D_{x}\left(q_{n+1, k}\right)=(-1)^{n} \operatorname{Res}\left(q_{n+1, k}, q_{n+1, k}^{\prime}\right),
$$

and by (4.5),

$$
\operatorname{Res}\left(q_{n+1, k}, x q_{n+1, k}^{\prime}\right)=\operatorname{Res}\left(q_{n+1, k}, x\right) \operatorname{Res}\left(q_{n+1, k}, q_{n+1, k}^{\prime}\right) .
$$

With (4.4) and (4.3) we get

$$
\operatorname{Res}\left(q_{n+1, k}, x\right)=\operatorname{Res}\left(x, q_{n+1, k}\right)=q_{n+1, k}(0)=1,
$$

so that

$$
D_{x}\left(q_{n+1, k}\right)=(-1)^{n} \operatorname{Res}\left(q_{n+1, k}, x q_{n+1, k}^{\prime}\right) .
$$


By differentiating (2.9) we obtain

$$
x q_{n+1, k}^{\prime}(x)=n q_{n+1, k}(x)+x^{n-1} V_{n}^{\prime}\left(\frac{1}{2}\left(x+\frac{1}{x}\right)\right) \frac{x^{2}-1}{2},
$$

and Lemma 4.1(b) gives

$$
\begin{aligned}
\operatorname{Res}\left(q_{n+1, k}, x q_{n+1, k}^{\prime}\right) & =\operatorname{Res}\left(q_{n+1, k}, x^{n-1} V_{n}^{\prime}\left(\frac{1}{2}\left(x+\frac{1}{x}\right)\right) \frac{x^{2}-1}{2}\right) \\
& =\operatorname{Res}\left(q_{n+1, k}, \frac{x^{2}-1}{2}\right) \operatorname{Res}\left(q_{n+1, k}, x^{n-1} V_{n}^{\prime}\left(\frac{1}{2}\left(x+\frac{1}{x}\right)\right)\right) .
\end{aligned}
$$

The first term on the right is

$$
\operatorname{Res}\left(q_{n+1, k}, \frac{x^{2}-1}{2}\right)=2^{-2 n}\left((n+1)^{2}-n^{2} k^{2}\right) ;
$$

this can be obtained by (5.12), with (4.5) and (4.6). To evaluate the second term on the right-hand side of (5.15), we follow the proof of Theorem 3, with $f(w)$ as before, and $g(w):=f^{\prime}(w)$. Then, in the notation of the proof of Theorem 3, we have

$$
\operatorname{Res}_{x}(F(x, 1), G(x, 1))=\operatorname{Res}_{x}\left(V_{n}(x), V_{n}^{\prime}(x)\right)=(-1)^{n} 2^{n} D_{x}\left(V_{n}\right),
$$

so that

$$
\operatorname{Res}_{z}\left(F\left(z^{2}+1,2 z\right), G\left(z^{2}+1,2 z\right)\right)=\left(2^{n} D_{x}\left(V_{n}\right)\right)^{2} 2^{2 n(n-1)}=2^{2 n^{2}} D_{x}\left(V_{n}\right)^{2}
$$

while on the other hand (see (5.1)), the left-hand side of this is

$$
2^{4 n(n-1)} \operatorname{Res}_{z}\left(q_{n+1, k}(z), z^{n-1} V_{n}^{\prime}\left(\frac{1}{2}\left(z+\frac{1}{z}\right)\right)\right) .
$$

This, combined with (5.14)-(5.17), gives (5.13), and the proof is complete.

Proof of Theorem 6. We begin the first part of the proof by setting, for fixed $y$, $f(w):=U_{n}(w)-y U_{n-1}(w)$. Then, by (2.8),

$$
2^{n} d_{n}(x, y)=(2 x)^{n} f\left(\frac{1+y x}{2 x}\right)=: F(1+y x, 2 x),
$$

with an analogous homogeneous polynomial $G$ to $f^{\prime}$. With the "interior" homogeneous polynomials

$$
H(x, u)=u+y x, \quad K(x, u)=2 x,
$$

we can once again continue as in the proof of Theorem 3 ; we leave the details to the reader.

For the second part we set $z:=(1+x y) / 2 x$. Then $y=2 z-\frac{1}{x}$, and with (2.7) in the form $2 z U_{n-1}(z)=U_{n}(z)+U_{n-2}(z)$ we rewrite (2.8) as

$$
\begin{aligned}
d_{n}(x, y) & =x^{n}\left[U_{n}(z)-\left(2 z-\frac{1}{x}\right) U_{n-1}(z)\right] \\
& =x^{n}\left[\frac{1}{x} U_{n-1}(z)-U_{n-2}(z)\right]=x^{n-1}\left[U_{n-1}(z)-x U_{n-2}(z)\right] .
\end{aligned}
$$

Finally, with both parts of Lemma 4.2 applied to this, we get

$$
\begin{aligned}
D_{y}\left(d_{n}(x, y)\right) & =\left(x^{n-1}\right)^{2(n-2)} D_{y}\left(U_{n-1}(z)-x U_{n-2}(z)\right) \\
& =x^{2(n-1)(n-2)}\left(\frac{1}{2}\right)^{(n-1)(n-2)} D_{y}\left(U_{n-1}(y)-x U_{n-2}(y)\right),
\end{aligned}
$$

and Theorem 4 then implies (B.6). 


\section{Proof of the main Result}

For the proof of Theorem 2 we use an interesting explicit expression due to Cygankova [5]. Let the relatively prime polynomials $f(x)=a_{0} x^{n}+a_{1} x^{n-1}+\cdots+a_{n}$ and $g(x)=b_{0} x^{n-1}+b_{1} x^{n-2}+\cdots+b_{n-1}$ be as in (4.1) with $m=n-1$, and suppose that the Euclidean algorithm

$$
\begin{aligned}
& f=H_{0} g+R_{0}, \\
& g=H_{1} R_{0}+R_{1},
\end{aligned}
$$

$$
\begin{aligned}
& R_{n-4}=H_{n-2} R_{n-3}+R_{n-2}, \\
& R_{n-3}=H_{n-1} R_{n-2}
\end{aligned}
$$

yields at every stage a quotient $H_{k}$ that is linear in $x$. Then we may write $H_{j}=$ $p_{j} x+q_{j}(j=0,1, \ldots, n-1)$. Also, $R_{j}$ is then a polynomial of degree $n-2-j$ $(j=0,1, \ldots, n-2)$. Under these conditions we have

Lemma 6.1 (Cygankova). With assumptions and notations as above, we have

$$
\operatorname{Res}(g, f)=\left(\frac{a_{0}}{p_{0}}\right)^{2 n-1} \prod_{j=1}^{n-1}\left(\frac{1}{p_{j}}\right)^{2 n-2 j-1} .
$$

A proof of this result can be obtained by iterating Lemma 4.1(a).

We now prove two more lemmas. We begin with a property of the polynomials $d_{n}(h, k)$ defined in (2.5); note that (2.8) is an equivalent definition.

Lemma 6.2. For $n \geq 1$ we have

$$
d_{n-1}(x, y) d_{n+1}(x, y)-d_{n}(x, y)^{2}=x^{2 n-1}(y-x) .
$$

Proof. We use the identities

$$
\begin{aligned}
U_{n-1}(z) U_{n+1}(z)-U_{n}(z)^{2} & =-1, \\
U_{n-2}(z) U_{n+1}(z)-U_{n-1}(z) U_{n}(z) & =-2 z,
\end{aligned}
$$

valid for $n \geq 1$, resp. $n \geq 2$. (6.4) is easy to prove by induction, using the recurrence relation (2.7); it can also be found, e.g., in [14], p. 40. (6.5) now follows immediately from (6.4), again using the recurrence relation.

For $n=1$, (6.3) can be verified by direct calculation. For $n \geq 2$ we use (2.8); then upon expanding, collecting terms according to powers of $y$, and using (6.4) and (6.5), the left-hand side of (6.3) becomes

$$
d_{n-1} d_{n+1}-d_{n}^{2}=x^{2 n}\left[-1-y\left(-2 \frac{1+x y}{2 x}\right)+y^{2}(-1)\right]=x^{2 n-1}(y-x) .
$$

The main ingredient in our proof of Theorem 2 is the following explicit evaluation of some of the terms in the Euclidean algorithm (6.1). For convenience, we define $R_{-1}=R_{-1}^{(n)}=g$.

Lemma 6.3. Let $f^{(n)}(x)=U_{n}(x)+k U_{n-1}(x)$ and $g^{(n)}(x)=U_{n-1}(x)+h U_{n-2}(x)$, and consider the Euclidean algorithm (6.1), with $f, g, H_{j}, R_{j}$ replaced by $f^{(n)}, g^{(n)}$, 
$H_{j}^{(n)}, R_{j}^{(n)}$. Then, setting $d_{j}=d_{j}(h, k)$, we have for $n \geq 2$,

$$
H_{j}^{(n)}=(-1)^{j}\left(\frac{d_{j}}{d_{j+1}}\right)^{2}\left(2 d_{j} d_{j+1} x+h^{2 j}(k-h)\right) \prod_{i=1}^{\lfloor j / 2\rfloor}\left(\frac{d_{j-2 i}}{d_{j+1-2 i}}\right)^{4}
$$

for $0 \leq j \leq n-2$, and

$$
\begin{aligned}
& H_{n-1}^{(n)}=(-1)^{n-1}\left(\frac{d_{n-1}}{d_{n}}\right)^{2}\left(2 d_{n-1} d_{n} x+d_{n-2} d_{n} h\right) \prod_{i=1}^{\lfloor(n-1) / 2\rfloor}\left(\frac{d_{n-1-2 i}}{d_{n-2 i}}\right)^{4}, \\
& R_{n-3}^{(n)}=(-1)^{(n-1)(n-2) / 2}\left(2 d_{n-1} x+h d_{n-2}\right) \prod_{i=1}^{\lfloor(n-2) / 2\rfloor}\left(\frac{d_{n-1-2 i}}{d_{n-2 i}}\right)^{2}, \\
& R_{n-2}^{(n)}=(-1)^{n(n-1) / 2} d_{n} \prod_{i=1}^{\lfloor(n-1) / 2\rfloor}\left(\frac{d_{n-2 i}}{d_{n+1-2 i}}\right)^{2} .
\end{aligned}
$$

Proof. We shall verify by induction on $n$ that all the rows of (6.1) are valid when each symbol has a superscript of $(n+1)$. For $n=2$ we have $f^{(2)}(x)=U_{2}(x)+$ $k U_{1}(x)=4 x^{2}+2 k x-1, g^{(2)}(x)=U_{1}(x)+h U_{0}(x)=2 x+h, H_{0}^{(2)}=2 x+(k-h)$, $R_{0}^{(2)}=-d_{2}=-1-h k+h^{2}$, and $H_{1}^{(2)}=-(2 x+h) / d_{2}$. We furthermore note that $R_{n-3}^{(n)}=g$ in this case, so we see that the two relevant rows in (6.1) are satisfied.

When $n=3$, we have $f^{(3)}(x)=8 x^{3}+4 k x^{2}-4 x-k, g^{(3)}(x)=4 x^{2}+2 h x-1$. Furthermore, (6.6) and (6.8) are $H_{0}^{(3)}=2 x+(k-h)$ and $R_{0}^{(3)}=-\left(2 d_{2} x+h\right)$, respectively, and with this we easily verify the first row in (6.1). Next, we have $H_{1}^{(3)}=-\left(2 d_{2} x+h^{2}(k-h)\right) / d_{2}^{2}, R_{1}^{(3)}=-d_{3} / d_{2}^{2}$, and this satisfies the second row of (6.1). Finally, (6.9) is $H_{2}^{(3)}=d_{2}^{2}\left(2 d_{2} x+h\right) / d_{3}$, and we can check that the last row in (6.1) is satisfied.

Now we suppose that the lemma holds for some $n-1$ and $n$, where $n \geq 3$. We use the fact that $H_{j}^{(n)}$ does not depend on $n$ as long as $j \leq n-2$, and subtract the lines of (6.1) for $n-1$ from $2 x$ times the corresponding lines of (6.1) for $n$ :

$$
\begin{aligned}
2 x f^{(n)}-f^{(n-1)} & =H_{0}^{(n+1)}\left(2 x g^{(n)}-g^{(n-1)}\right)+\left(2 x R_{0}^{(n)}-R_{0}^{(n-1)}\right), \\
2 x g^{(n)}-g^{(n-1)} & =H_{1}^{(n+1)}\left(2 x R_{0}^{(n)}-R_{0}^{(n-1)}\right)+\left(2 x R_{1}^{(n)}-R_{1}^{(n-1)}\right), \\
2 x R_{0}^{(n)}-R_{0}^{(n-1)} & =H_{2}^{(n+1)}\left(2 x R_{1}^{(n)}-R_{1}^{(n-1)}\right)+\left(2 x R_{2}^{(n)}-R_{2}^{(n-1)}\right),
\end{aligned}
$$

$$
\begin{aligned}
& 2 x R_{n-5}^{(n)}-R_{n-5}^{(n-1)}=H_{n-3}^{(n+1)}\left(2 x R_{n-4}^{(n)}-R_{n-4}^{(n-1)}\right)+\left(2 x R_{n-3}^{(n)}-R_{n-3}^{(n-1)}\right), \\
& 2 x R_{n-4}^{(n)}-R_{n-4}^{(n-1)}=H_{n-2}^{(n+1)} 2 x R_{n-3}^{(n)}-H_{n-2}^{(n-1)} R_{n-3}^{(n-1)}+2 x R_{n-2}^{(n)}, \\
& 2 x R_{n-3}^{(n)}-R_{n-3}^{(n-1)}=H_{n-1}^{(n)} 2 x R_{n-2}^{(n)}-R_{n-3}^{(n-1)} .
\end{aligned}
$$

By definition and (2.7) we have

$$
f^{(n+1)}=2 x f^{(n)}-f^{(n-1)}, \quad g^{(n+1)}=2 x g^{(n)}-g^{(n-1)},
$$

and we set, for $0 \leq j \leq n-3$,

$$
R_{j}^{(n+1)}:=2 x R_{j}^{(n)}-R_{j}^{(n-1)} .
$$


This gives the first $n-2$ rows of the Euclidean algorithm for $f^{(n+1)}$ and $g^{(n+1)}$. For (6.10) to be completely consistent with (6.1) for $n+1$, we still have to verify the equations (6.13), (6.14), and (6.15) below, in which $H_{n}^{(n+1)}, R_{n-2}^{(n+1)}$, and $R_{n-1}^{(n+1)}$ are given by (6.7)-(6.9) with $n$ replaced by $n+1$. In other words, we have to verify that the penultimate equation of (6.10) has the form

$$
\begin{aligned}
H_{n-2}^{(n+1)} 2 x R_{n-3}^{(n)} & -H_{n-2}^{(n-1)} R_{n-3}^{(n-1)}+2 x R_{n-2}^{(n)} \\
& =H_{n-2}^{(n+1)}\left(2 x R_{n-3}^{(n)}-R_{n-3}^{(n-1)}\right)+R_{n-2}^{(n+1)},
\end{aligned}
$$

that the final equation of (6.10) has the form

$$
H_{n-1}^{(n)} 2 x R_{n-2}^{(n)}-R_{n-3}^{(n-1)}=H_{n-1}^{(n+1)} R_{n-2}^{(n+1)}+R_{n-1}^{(n+1)}
$$

and finally, for the last row of the $f^{(n+1)}, g^{(n+1)}$ algorithm, i.e., the last row of (6.1), that we have

$$
R_{n-2}^{(n+1)}=H_{n}^{(n+1)} R_{n-1}^{(n+1)}
$$

We begin with (6.13); it simplifies to

$$
R_{n-3}^{(n-1)}\left(H_{n-2}^{(n+1)}-H_{n-2}^{(n-1)}\right)=R_{n-2}^{(n+1)}-2 x R_{n-2}^{(n)} .
$$

We use (6.6) - 6.9), and after canceling all the obvious terms we have

$$
(-1)^{(n-1)(n-2) / 2}(-1)^{n}\left[h^{2 n-5}(k-h)-d_{n-3} d_{n-1}\right]=(-1)^{n(n-1) / 2} d_{n-2}^{2} .
$$

The powers of -1 all combine to -1 , and by Lemma 6.2 the terms in brackets on the left-hand side equal $-d_{n-2}^{2}$; this verifies (6.13). Similarly, 6.14) can be rewritten as

$$
\begin{aligned}
d_{n}^{2}\left[2 d_{n-1} x+d_{n-2} h\right] & 2 x-d_{n-1} d_{n}^{2} \\
= & {\left[d_{n-1} d_{n} 2 x+h^{2 n-2}(k-h)\right]\left[2 d_{n} x+h d_{n-1}\right]-d_{n+1} d_{n-1}^{2} . }
\end{aligned}
$$

The coefficients of $x^{2}$ on both sides are immediately seen to be equal, and the coefficients of $x$ and the constant coefficients are equal by Lemma 6.2. Finally, (6.15) is easily verified with (6.7)-(6.9). This completes the induction, and we have shown that $f^{(n)}(x)$ and $g^{(n)}(x)$ lead to the Euclidean algorithm [6.1).

We are now ready to prove Theorem 2 . We use (6.2) with $g=g^{(n)}, f=f^{(n)}$ as defined in Lemma 6.3. Since $U_{n}(x)=2^{n} x^{n}+\ldots$, we have $a_{0}=2^{n}$. Let

$$
P_{n}:=\prod_{j=0}^{n-1}\left(\frac{1}{p_{j}}\right)^{2 n-2 j-1} .
$$

We show by induction that, for $n \geq 1$,

$$
P_{n}=\frac{(-1)^{\frac{n(n-1)}{2}}}{2^{n^{2}}} d_{n} .
$$

Indeed, we have $P_{1}=1 / p_{0}=1 / 2=(1 / 2) d_{1}$. Next, suppose that 6.17) holds for some $n \geq 1$. Then, by (6.16),

$$
P_{n+1}=\frac{P_{n}}{p_{n}} \prod_{j=0}^{n-1} p_{j}^{-2} .
$$


By (6.6) and (6.7) we have, independently of $n$,

$$
p_{j}=(-1)^{j} 2 \frac{d_{j}^{3}}{d_{j+1}} \prod_{i=1}^{\lfloor j / 2\rfloor}\left(\frac{d_{j-2 i}}{d_{j+1-2 i}}\right)^{4} .
$$

Upon applying the operational identity

$$
\prod_{j=0}^{n-1} \prod_{i=1}^{\lfloor j / 2\rfloor}=\prod_{i=1}^{\lfloor n / 2\rfloor} \prod_{j=2 i}^{n-1}
$$

we find from (6.19), thanks to the telescoping of a product, that

$$
\prod_{j=0}^{n-1} p_{j}=(-1)^{\frac{n(n-1)}{2}} 2^{n} \frac{1}{d_{n}} \prod_{i=0}^{\left\lfloor\frac{n-2}{2}\right\rfloor}\left(\frac{d_{n-1-2 i}}{d_{n-2-2 i}}\right)^{2},
$$

and this combined with (6.18), (6.17), and (6.19) gives

$$
P_{n+1}=\frac{(-1)^{\frac{(n+1) n}{2}}}{2^{(n+1)^{2}}} d_{n+1}
$$

which completes the proof by induction.

Finally, (6.17), (6.16), and (6.2) together prove (2.10), and we are done. Note that, by (4.4), interchanging the order of the arguments in the resultants in (2.10) and (6.2) does not change the sign in this case.

\section{Some CONSEQUences}

We first note that Lemma 6.3 immediately gives a finite continued fraction expansion of $f^{(n)}(x) / g^{(n)}(x)$. Interesting special cases could be derived by particular choices of the parameters $h, k$ and the variable $x$. There are well-known close connections between orthogonal polynomials and continued fractions (see, e.g., [17]); we will not pursue this further in this paper.

We now derive some easy special cases of Theorem 2. It is clear from (2.8) that $y=0$ leads to a simplification.

Corollary 7.1. For $n \geq 2$ we have

$$
\operatorname{Res}\left(U_{n}(x), U_{n-1}(x)+h U_{n-2}(x)\right)=(-1)^{\frac{n(n-1)}{2}} 2^{n(n-1)} h^{n} U_{n}\left(\frac{1}{2 h}\right),
$$

and, in particular,

$$
\operatorname{Res}\left(U_{n}(x), U_{n-1}(x)\right)=(-1)^{\frac{n(n-1)}{2}} 2^{n(n-1)} .
$$

Next we use the well-known relation connecting the Chebyshev polynomials of both kinds, namely

$$
T_{n}(z)=U_{n}(z)-z U_{n-1}(z)
$$

(see, e.g., [1, p. 777). Considering (2.8), this suggests that the case $k=(1+h k) / 2 h$, which is equivalent to $h k=1$, plays a special role. Indeed, we easily obtain

Corollary 7.2. For $n \geq 2$ we have

$$
\operatorname{Res}\left(U_{n}(x)+\frac{1}{h} U_{n-1}(x), U_{n-1}(x)+h U_{n-2}(x)\right)=(-1)^{\frac{n(n-1)}{2}} 2^{n(n-1)} h^{n} T_{n}\left(\frac{1}{h}\right) .
$$


Next, we observe that

$$
D_{x}\left(U_{n}(x)\right)=2^{n^{2}}(n+1)^{n-2}
$$

is an immediate consequence of (3.2) and (3.9). Finally, as a curiosity, both the Fibonacci numbers and the Lucas numbers can be obtained as resultants. Indeed, if we use the well-known relations

$$
F_{n+1}=(-i)^{n} U_{n}\left(\frac{i}{2}\right), \quad L_{n}=\frac{1}{2} i^{n} T_{n}\left(\frac{i}{2}\right)
$$

(see, e.g., [14], pp. 61 f.), then Corollaries 7.1 and 7.2 immediately imply the following formulas.

Corollary 7.3. For $n \geq 2$ we have

$$
\begin{aligned}
\operatorname{Res}\left(U_{n}(x), U_{n-1}(x)-i U_{n-2}(x)\right) & =(-1)^{\frac{n(n-1)}{2}} 2^{n(n-1)} F_{n+1}, \\
\operatorname{Res}\left(U_{n}(x)+\frac{i}{2} U_{n-1}(x), U_{n-1}(x)-2 i U_{n-2}(x)\right) & =(-1)^{\frac{n(n+1)}{2}} 2^{n^{2}+1} L_{n} .
\end{aligned}
$$

\section{REFERENCES}

[1] M. Abramowitz and I.A. Stegun, Handbook of Mathematical Functions, National Bureau of Standards, 1964. MR0208798 (34:8607)

[2] G.E. Andrews, R. Askey, and R. Roy, Special Functions, Cambridge University Press, 1999. MR.1688958(2000g:33001)

[3] T. M. Apostol, Resultants of cyclotomic polynomials, Proc. Amer. Math. Soc. 24 (1970), 457-462. MR 0251010 (40:4241)

[4] T. M. Apostol, The resultants of the cyclotomic polynomials $F_{m}(a x)$ and $F_{n}(b x)$, Math. Comp. 29 (1975), 1-6. MR0366801 (51:3047)

[5] O. I. Cygankova, Formulae for calculating the discriminants of Jacobi, Laguerre and Hermite polynomials (Russian), Izv. Vysš. Učebn. Zaved. Matematika 1962 (1962), no. 4 (29), 170172. MR0138805 (25:2248)

[6] D. Drucker and G. Greenfield, On the discriminant of a trinomial, Lin. Alg. Appl. 62 (1984), 105-112. MR0761061 (86c:12005)

[7] I. M. Gel'fand, M. M. Kapranov, and A. V. Zelevinsky, Discriminants, Resultants, and Multidimensional Determinants, Birkhäuser, Boston, 1994. MR1264417 (95e:14045)

[8] M.E.H. Ismail, Discriminants and functions of the second kind of orthogonal polynomials, Result. Math. 34 (1998), 132-149. MR.1635590 (99g:42032)

[9] S. McCallum, Factors of iterated resultants and discriminants, J. Symbolic Computation 27 (1999), 367-385. MR1681345 (2000b:13034)

[10] J. H. McKay and S. Sui-Sheng Wang, A chain rule for the resultant of two polynomials, Arch. Math. (Basel) 53 (1989), 347-351. MF1015998 (90h:12006)

[11] J. H. McKay and S. Sui-Sheng Wang, A chain rule for the resultant of two homogeneous polynomials, Arch. Math. (Basel) 56 (1991), 352-361. MR1094422 (92a:12006)

[12] M. Pohst and H. Zassenhaus, Algorithmic Algebraic Number Theory, Cambridge University Press, Cambridge, 1989. MR1033013 (92b:11074)

[13] G. Rado, Die Diskriminante der allgemeinen Kreisteilungsgleichung, J. Reine Angew. Math. 131 (1906), 49-55.

[14] T. J. Rivlin, Chebyshev Polynomials, second edition, Wiley, New York, 1990. MR1060735 (92a:41016)

[15] G. Shabat and A. Zvonkin, Plane trees and algebraic numbers. Jerusalem combinatorics '93, 233-275, Contemp. Math., 178, Amer. Math. Soc., Providence, RI, 1994. MR1310587 (96d:14028)

[16] R. Swan, Factorization of polynomials over finite fields, Pacific J. Math. 12 (1962), 10991106. MR0144891 (26:2432) 
[17] G. Szegö, Orthogonal Polynomials, fourth edition, American Mathematical Society, Providence, Rhode Island, 1975. MR0372517 (51:8724)

[18] B. L. van der Waerden, Modern Algebra, Vol. 1, Ungar, New York, 1949. MR0029363 (10:587b)

Department of Mathematics and Statistics, Dalhousie University, Halifax, Nova ScoTiA, CANADA B3H 3J5

E-mail address: dilcher@mathstat.dal.ca

Department of Mathematics, University of Illinois, 1409 West Green Street, UrBANA, ILLINOIS 61801

E-mail address: stolarsk@math.uiuc.edu 